\title{
Quarkonium suppression in heavy-ion collisions from coherent energy loss in cold nuclear matter
}

\author{
François Arleo, ${ }^{a, b}$ Stéphane Peigné ${ }^{c}$ \\ ${ }^{a}$ Laboratoire Leprince-Ringuet (LLR), École polytechnique, \\ CNRS/IN2P3 91128 Palaiseau, France \\ ${ }^{b}$ Laboratoire d'Annecy-le-Vieux de Physique Théorique (LAPTh) \\ UMR5108, Université de Savoie, CNRS, BP 110, 74941 Annecy-le-Vieux cedex, France \\ ${ }^{c}$ SUBATECH, UMR 6457, Université de Nantes, Ecole des Mines de Nantes, IN2P3/CNRS \\ 4 rue Alfred Kastler, 44307 Nantes cedex 3, France \\ E-mail: francois.arleo@cern.ch, peigne@subatech.in2p3.fr
}

\begin{abstract}
The effect of parton energy loss in cold nuclear matter on the suppression of quarkonia $(\mathrm{J} / \psi, \Upsilon)$ in heavy-ion collisions is investigated, by extrapolating a model based on coherent radiative energy loss recently shown to describe successfully $\mathrm{J} / \psi$ and $\Upsilon$ suppression in proton-nucleus collisions. Model predictions in heavy-ion collisions at RHIC ( $\mathrm{Au}-\mathrm{Au}, \mathrm{Cu}-\mathrm{Cu}$, and $\mathrm{Cu}-\mathrm{Au})$ and $\mathrm{LHC}(\mathrm{Pb}-\mathrm{Pb})$ show a sizable suppression arising from the sole effect of energy loss in cold matter. This effect should thus be considered in order to get a reliable baseline for cold nuclear matter effects in quarkonium suppression in heavy-ion collisions, in view of disentangling hot from cold nuclear effects.
\end{abstract}

KEYwords: Phenomenological Models, Heavy Ion Phenomenology

ARXIV EPRINT: 1407.5054 


\section{Contents}

1 Introduction and summary 1

2 Model for quarkonium suppression in nuclear collisions 3

2.1 Proton-nucleus collisions 3

2.2 Nucleus-nucleus collisions 4

2.3 Medium length in the Glauber model 6

$\begin{array}{lll}2.4 & \text { A simple approximation } & 7\end{array}$

2.5 Computing uncertainties 9

$\begin{array}{ll}2.6 & \text { Range of validity } \\ & 10\end{array}$

$\begin{array}{lll}3 & \text { RHIC } & 10\end{array}$

$\begin{array}{lll}3.1 & \text { Rapidity dependence } & 10\end{array}$

$\begin{array}{ll}3.2 \text { Centrality dependence } & 11\end{array}$

$\begin{array}{lll}4 & \text { LHC } & 13\end{array}$

$\begin{array}{ll}4.1 \text { Rapidity dependence } & 13\end{array}$

$\begin{array}{lll}4.2 & \text { Centrality dependence } & 14\end{array}$

$\begin{array}{lll}5 & \text { Discussion } & 15\end{array}$

$\begin{array}{ll}\text { A Quenching weight } & 17\end{array}$

\section{Introduction and summary}

Quarkonium production in heavy-ion (A-A) collisions is a widely discussed observable, expected to be highly sensitive to the presence of a hot medium. However, predicting quarkonium production rates in A-A collisions is a difficult task, due to various competing effects, such as quarkonium suppression from Debye screening in a hot medium [1] and enhancement due to recombination processes at high energy $[2,3]$. In order to interpret reliably the heavy-ion measurements, accurate baseline predictions assuming only cold nuclear effects are needed.

Until quite recently, most of the phenomenological approaches assumed either nuclear absorption, nuclear parton distribution function (nPDF) or saturation effects to be responsible for $\mathrm{J} / \psi$ and $\Upsilon$ suppression in proton-nucleus (p-A) collisions. In a series of papers, however, we argued that medium-induced parton energy loss in cold nuclear matter could play a decisive role in the suppression of $\mathrm{J} / \psi$ and $\Upsilon$ states (denoted as $\psi$ in the following) in p-A collisions [4-7]. Quite remarkably, all available $\psi$ suppression measurements from fixed-target experiments (SPS, HERA, FNAL) to RHIC could be described within a simple model and on a broad kinematical range in rapidity [6] and transverse momentum [7]. 
What is more, predictions in $\mathrm{p}-\mathrm{Pb}$ collisions at the LHC proved in excellent agreement with ALICE [8] and LHCb [9] data.

We emphasize that this successful description of the $\psi$ suppression data at various collision energies can be obtained, with a very good $\chi^{2} /$ ndf, without including nPDF effects [6]. On the contrary, $\mathrm{nPDF} /$ shadowing effects alone cannot achieve such a global description, in particular they fail to describe the shape of $\psi$ suppression as a function of $x_{\mathrm{F}}$ (or of the rapidity $y$ ), at both fixed-target and collider energies. ${ }^{1}$ This suggests switching the way to apprehend cold nuclear effects in quarkonium suppression, namely, to consider parton energy loss as the leading effect. Shadowing/nPDF effects may affect the magnitude of $\psi$ suppression but not so much the shape ${ }^{2}$ and might thus be viewed as 'corrections' which do not change the qualitative picture of $\psi$ suppression obtained with parton energy loss alone. ${ }^{3}$ Let us also mention that medium-induced parton energy loss is as fundamental as nPDFs, and could actually apply more generally. As a matter of fact, it should also play a role in processes breaking QCD factorization, for which a description in terms of nPDFs alone would be unfounded.

The agreement of the model used in refs. [5-7] with $\psi$ suppression data in p-A collisions originates mainly from the parametric behavior $\Delta E \propto E$ (where $E$ is the $\psi$ energy) of the medium-induced radiative parton energy loss. In particular this behavior is essential to describe the increase of $\psi$ suppression with increasing rapidity. This parametric law arises when a fast incoming color charge crosses the target nucleus and is scattered to small angle (in the target rest frame). It is thus expected to hold in quarkonium hadroproduction, where typically a high-energy gluon from the projectile proton is scattered to a compact color octet heavy $Q \bar{Q}$ pair [4]. The radiative loss $\Delta E \propto E$ originates from gluon radiation which is fully coherent over the size $L$ of the nucleus. The parametric dependence (in $E, L$ and the mass $M$ of the produced compact color state) of the coherent radiation spectrum and associated average loss $\Delta E$ was first derived in [4], and recently reviewed in [11] in a fully defined theoretical setup. Note that the same coherent, medium-induced radiation spectrum arises in the production of a single forward particle $[4,11]$ and in forward dijet production $[12,13]$, suggesting the broad relevance of coherent energy loss in hard forward p-A processes.

In the present study we extrapolate the model of refs. [5-7] to nucleus-nucleus collisions. To illustrate the main idea, consider the production of a compact color octet $Q \bar{Q}$ pair through gluon-gluon fusion, at mid-rapidity (and low transverse momentum, $p_{\perp} \lesssim M$ ) in the nucleon-nucleon c.m. frame of some A-B collision. In the rest frame of the nucleus $\mathrm{B}$, the $Q \bar{Q}$ pair is produced at large (positive) rapidity from the incoming fast gluon of

\footnotetext{
${ }^{1}$ The difficulty for nPDF effects to produce a correct parametric dependence of $\psi$ suppression is wellknown: if shadowing effects would play a dominant role for $\psi$ nuclear suppression, the latter should scale in the target momentum fraction, $x_{2}$. However a drastic violation of $x_{2}$-scaling is observed when comparing the $\psi$ suppression data at various collision energies, as first noted in [10].

${ }^{2}$ The effect of nPDFs on the magnitude of $\psi$ suppression in $\mathrm{p}-\mathrm{A}$ collisions is minor at fixed-target, and sizable at collider energies [6]. Regarding the shape, nPDF effects lead to a flatter rapidity (or, $x_{\mathrm{F}}$ ) dependence of $\psi$ suppression in p-A collisions, at all $\sqrt{s}$, as compared to energy loss effects [6].

${ }^{3}$ One exception, however, is the case of the total $\psi$ production cross section, which should be sensitive to nPDF effects but not to energy loss effects (which only affect the $y$ and $p_{\perp}$ differential cross sections).
} 
the 'projectile' A. This leads to a suppression of the $\psi$ production rate due to coherent energy loss induced by rescatterings in $\mathrm{B}$, as shown in [6] in the case of $\mathrm{p}-\mathrm{B}$ collisions. Analogously, when viewed in the rest frame of $\mathrm{A}, \psi$ production at large (negative) rapidity must be affected by the coherent energy loss induced by rescatterings in $\mathrm{A}$, leading to an additional $\psi$ suppression at $y=0$ in the c.m. frame. ${ }^{4}$

The goal of the present study is to provide baseline predictions, based on coherent energy loss through cold nuclear matter, for the rapidity ${ }^{5}$ and centrality dependence of $\mathrm{J} / \psi$ and $\Upsilon$ suppression in heavy-ion collisions at RHIC and LHC. The model is first generalized to deal with nucleus-nucleus collisions in section 2. The predictions at RHIC and LHC are given in sections 3 and 4, where they are also compared to the heavy-ion data. In the final discussion, section 5, we argue that the observed discrepancies between the baseline predictions and the data are qualitatively consistent with the presence of additional hot suppression effects in heavy-ion collisions, and of recombination processes in the specific case of $\mathrm{J} / \psi$ production at the LHC. Although the present study focusses on parton energy loss, we also shortly discuss nPDF effects in section 5. Using different nPDF sets, we roughly estimate $\mathrm{J} / \psi$ suppression due to $\mathrm{nPDF}$ effects alone, and observe that it never exceeds the strength of the suppression assuming only coherent energy loss. In particular, at the LHC and at large enough rapidity, the effect of energy loss on $\mathrm{J} / \psi$ suppression dominates over the effect of nPDFs.

\section{Model for quarkonium suppression in nuclear collisions}

\subsection{Proton-nucleus collisions}

We briefly remind in this section the basics of the model based on coherent energy loss used to describe $\psi$ suppression measured in proton-nucleus collisions. The single differential p-B production cross section as a function of the $\psi$ energy reads [6]

$$
\frac{1}{B} \frac{\mathrm{d} \sigma_{\mathrm{pB}}^{\psi}}{\mathrm{d} E_{\mathrm{B}}}\left(E_{\mathrm{B}}\right)=\int_{0}^{\varepsilon^{\max }} \mathrm{d} \varepsilon_{\mathrm{B}} \mathcal{P}\left(\varepsilon_{\mathrm{B}}, E_{\mathrm{B}}, \ell_{\mathrm{B}}^{2}\right) \frac{\mathrm{d} \sigma_{\mathrm{pp}}^{\psi}}{\mathrm{d} E_{\mathrm{B}}}\left(E_{\mathrm{B}}+\varepsilon_{\mathrm{B}}\right)
$$

where $E_{\mathrm{B}}$ (respectively, $\varepsilon_{\mathrm{B}}$ ) is the energy (respectively, energy loss) of the $Q \bar{Q}$ pair in the rest frame of the nucleus $\mathrm{B}$. The upper limit on the energy loss is $\varepsilon^{\max }=\min \left(E_{\mathrm{B}}, E_{\mathrm{p}}-E_{\mathrm{B}}\right)$, where $E_{\mathrm{p}}$ is the beam energy in that frame, and the p-p production cross section is given by a fit to data. $\mathcal{P}$ denotes the energy loss probability distribution, or quenching weight.

The quenching weight is related to the medium-induced, coherent radiation spectrum $\mathrm{d} I / \mathrm{d} \varepsilon$ given in [6] (and earlier in [4]), which is a very good approximation to the exact spectrum computed to all orders in the opacity expansion [11]. For convenience the explicit expression of $\mathcal{P}$ is quoted in appendix A. It depends on the accumulated transverse momentum transfer $\ell_{\mathrm{B}}=\sqrt{\hat{q} L_{\mathrm{B}}}$ (assumed to satisfy $\left.\ell_{\mathrm{B}} \ll M_{\perp}\right)$ due to soft rescatterings

\footnotetext{
${ }^{4}$ We expect the two effects to add incoherently, because rescatterings in A and B induce gluon radiation spectra which populate different regions of phase space, see section 2.2 and figure 1.

${ }^{5}$ The transverse momentum dependence of quarkonium suppression in heavy-ion collisions will be addressed in a separate study.
} 
in nucleus B, where $L_{\mathrm{B}}$ is the path-length discussed in section 2.3 and $\hat{q}$ the transport coefficient in cold nuclear matter. More precisely [6],

$$
\hat{q} \equiv \hat{q}_{0}\left[\frac{10^{-2}}{\min \left(x_{0}, x_{2}\right)}\right]^{0.3} ; \quad x_{0} \equiv \frac{1}{2 m_{\mathrm{p}} L_{\mathrm{B}}} ; \quad x_{2} \equiv \frac{M_{\perp}}{\sqrt{s}} e^{-y},
$$

where $y$ is the $\psi$ rapidity in the center-of-mass frame of the proton-nucleon collision (of energy $\sqrt{s} \simeq \sqrt{2 m_{\mathrm{p}} E_{\mathrm{p}}}$, with $m_{\mathrm{p}}$ the proton mass), and $M_{\perp}=\left(M^{2}+p_{\perp}^{2}\right)^{\frac{1}{2}}$ is the transverse mass of the $Q \bar{Q}$ pair. In the present paper, we consider quarkonium production integrated over $p_{\perp}$ (and thus dominated by typical values $p_{\perp} \lesssim M$ ) for which using $2 \rightarrow 1$ kinematics for the partonic subprocess is a reasonable simplifying assumption. The value of $\hat{q}_{0}$ used in this analysis and its uncertainty are discussed in section 2.5. For clarity, the dependence of $\hat{q}$ on the medium size $L$ (through the value of $x_{0}$, see (2.2)) will be implicit in the following.

In view of generalizing the model to A-B collisions in the next section, where the projectile and target play symmetric roles, it is convenient to change variable from $E_{\mathrm{B}}$ to the (proton-nucleon) c.m. frame rapidity $y$, using

$$
E_{\mathrm{B}}=E_{\mathrm{p}} \frac{M_{\perp}}{\sqrt{s}} e^{y} \equiv E(y) .
$$

From (2.1) we obtain

$$
\frac{1}{B} \frac{\mathrm{d} \sigma_{\mathrm{pB}}^{\psi}}{\mathrm{d} y}=\int_{0}^{\varepsilon^{\max }(y)} \mathrm{d} \varepsilon_{\mathrm{B}} \mathcal{P}\left(\varepsilon_{\mathrm{B}}, E(y), \hat{q}(y) L_{\mathrm{B}}\right)\left[\frac{E(y)}{E(y)+\varepsilon_{\mathrm{B}}}\right] \frac{\mathrm{d} \sigma_{\mathrm{pp}}^{\psi}}{\mathrm{d} y}\left(y\left(E(y)+\varepsilon_{\mathrm{B}}\right)\right),
$$

where $\varepsilon^{\max }(y)=\min \left(E(y), E_{\mathrm{p}}-E(y)\right)$. We now change the integration variable and express the energy loss $\varepsilon_{\mathrm{B}}$ in terms of a shift in rapidity, $\delta y_{\mathrm{B}}$, defined as

$$
E(y)+\varepsilon_{\mathrm{B}} \equiv E\left(y+\delta y_{\mathrm{B}}\right)=E(y) e^{\delta y_{\mathrm{B}}} \Leftrightarrow \delta y_{\mathrm{B}}=\ln \left(1+\frac{\varepsilon_{\mathrm{B}}}{E(y)}\right) .
$$

Using the fact that the quenching weight is a scaling function of the variable $x=\varepsilon / E$, namely $E \mathcal{P}\left(\varepsilon, E, \ell^{2}\right)=\hat{\mathcal{P}}\left(x \equiv \varepsilon / E, \ell^{2}\right)$, we can rewrite $(2.4)$ as

$$
\frac{1}{B} \frac{\mathrm{d} \sigma_{\mathrm{pB}}^{\psi}}{\mathrm{d} y}(y)=\int_{0}^{\delta y^{\max }(y)} \mathrm{d} \delta y_{\mathrm{B}} \hat{\mathcal{P}}\left(e^{\delta y_{\mathrm{B}}}-1, \hat{q}(y) L_{\mathrm{B}}\right) \frac{\mathrm{d} \sigma_{\mathrm{pp}}^{\psi}}{\mathrm{d} y}\left(y+\delta y_{\mathrm{B}}\right) .
$$

Here $\delta y^{\max }(y)=\min \left(\ln 2, y_{\max }-y\right)$, with $y_{\max }=\ln \left(\sqrt{s} / M_{\perp}\right)$ the maximal $\psi$ rapidity (in the proton-nucleon c.m. frame) allowed by our kinematics. ${ }^{6}$

The expression (2.6), together with the explicit form of $\hat{\mathcal{P}}$ given in appendix A (see (A.5)), was used in $[6,7]$ to study $\psi$ nuclear suppression in proton-nucleus collisions.

\subsection{Nucleus-nucleus collisions}

Let us now consider the more complicated case of quarkonium production in nucleusnucleus collisions. In a generic A-B collision both incoming partons, respectively from the

\footnotetext{
${ }^{6}$ For $\mathrm{J} / \psi$ production, taking $M=3 \mathrm{GeV}$ for the $c \bar{c}$ pair and $p_{\perp}=1 \mathrm{GeV}$, we obtain $y_{\max } \simeq 4.1$ at RHIC $(\sqrt{s}=200 \mathrm{GeV})$ and $y_{\max } \simeq 6.8$ at $\mathrm{LHC}(\sqrt{s}=2.76 \mathrm{TeV})$.
} 


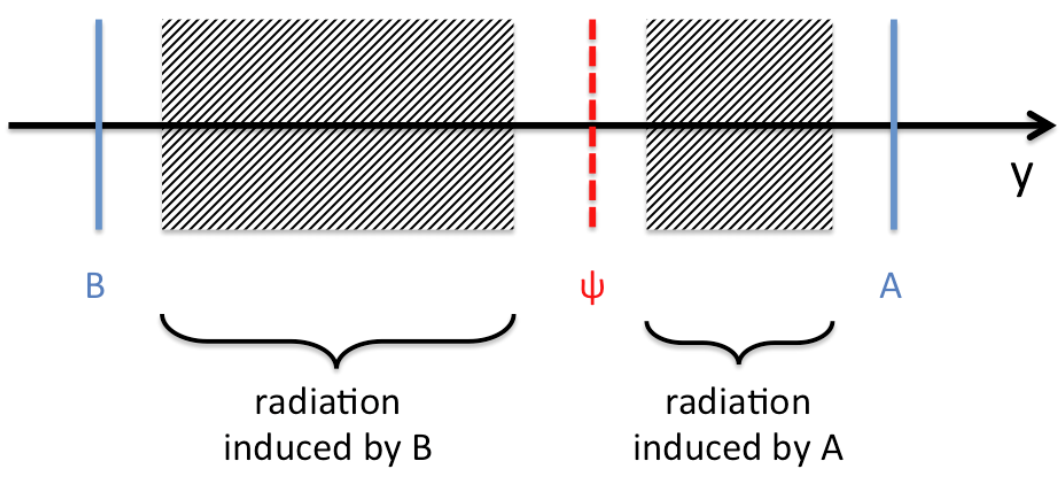

Figure 1. Sketch of the rapidity regions populated by medium-induced radiation in an A-B collision. The 'target' B and 'projectile' A move with respectively negative and positive rapidities.

'projectile' nucleus A and the 'target' nucleus B, might suffer multiple scattering in the nucleus $\mathrm{B}$ and $\mathrm{A}$, respectively. Consequently, gluon radiation off both partons can interfere with that of the final state particle (here, the compact color octet $Q \bar{Q}$ pair), making a priori difficult the calculation of the medium-induced gluon spectrum in the collision of two heavy ions.

However, consider gluon radiation induced by rescattering in nucleus B. The mediuminduced, coherent gluon spectrum is found in $[4,11]$ to arise from the logarithmic $k_{\perp}$ domain

$$
\frac{\omega}{E_{\mathrm{B}}} M_{\perp} \ll k_{\perp} \ll \ell_{\mathrm{B}}
$$

where $\omega$ and $k_{\perp}$ denote the radiated gluon energy (in the B rest frame) and transverse momentum, and $\ell_{\mathrm{B}} \ll M_{\perp}$ is the typical transverse broadening in nucleus B already introduced in section 2.1. ${ }^{7}$

Introducing the rapidities of the $\psi$ state and of the radiated gluon in the $\mathrm{B}$ frame,

$$
y_{\mathrm{B}}^{\psi}=\frac{1}{2} \ln \left(\frac{E_{\mathrm{B}}+p_{\mathrm{B}}^{z}}{E_{\mathrm{B}}-p_{\mathrm{B}}^{z}}\right) \simeq \ln \left(\frac{2 E_{\mathrm{B}}}{M_{\perp}}\right) ; y_{\mathrm{B}}^{g}=\frac{1}{2} \ln \left(\frac{\omega+k^{z}}{\omega-k^{z}}\right) \simeq \ln \left(\frac{2 \omega}{k_{\perp}}\right),
$$

the leftmost inequality in (2.7) becomes

$$
\frac{\omega}{k_{\perp}} \ll \frac{E_{\mathrm{B}}}{M_{\perp}} \Rightarrow \exp \left(y_{\mathrm{B}}^{g}\right) \ll \exp \left(y_{\mathrm{B}}^{\psi}\right) \Rightarrow y_{\mathrm{B}}^{g}<y_{\mathrm{B}}^{\psi} .
$$

The latter inequality must hold in all longitudinally boosted frames, namely, $y^{g}<y^{\psi}$. Thus, the medium-induced radiation associated to rescattering in B populates the region of rapidities smaller than the $\psi$ rapidity. Similarly, the induced radiation associated to rescattering in A populates $y^{g}>y^{\psi}$. This is illustrated in figure 1 .

\footnotetext{
${ }^{7}$ Obviously the logarithmic range (2.7) only appears when $\omega \ll E_{\mathrm{B}} \ell_{\mathrm{B}} / M_{\perp}$, i.e., when $\omega$ is much smaller than the typical $\omega$ contributing to the average energy loss $\Delta E$ [4]. In the present study we focus on such $\omega$ values, which can be checked to dominate in the convolution (2.1) (where $\varepsilon_{\mathrm{B}}=\omega$ ), due to the fast decrease of the p-p cross section with increasing rapidity [6].
} 
Since gluon radiation induced by rescattering in nuclei A and $\mathrm{B}$ occurs in distinct regions of phase space, it can be combined in a probabilistic manner as follows. We first express the $\psi$ production cross section in A-B simply as a function of that in A-p collisions using (2.6)

$$
\frac{1}{A B} \frac{\mathrm{d} \sigma_{\mathrm{AB}}^{\psi}}{\mathrm{d} y}(y)=\int_{0}^{\delta y^{\max (y)}} \mathrm{d} \delta y_{\mathrm{B}} \hat{\mathcal{P}}\left(e^{\delta y_{\mathrm{B}}}-1, \hat{q}(y) L_{\mathrm{B}}\right) \frac{1}{A} \frac{\mathrm{d} \sigma_{\mathrm{Ap}}^{\psi}}{\mathrm{d} y}\left(y+\delta y_{\mathrm{B}}\right) .
$$

Then, using again (2.6) to express the A-p cross section as a function of that in p-p collisions, one obtains ${ }^{8}$

$$
\frac{1}{A B} \frac{\mathrm{d} \sigma_{\mathrm{AB}}^{\psi}}{\mathrm{d} y}(y)=\int_{0}^{\delta y^{\max }(y)} \mathrm{d} \delta y_{\mathrm{B}} \hat{\mathcal{P}}\left(x_{\mathrm{B}}, \hat{q}(y) L_{\mathrm{B}}\right) \int_{0}^{\delta y^{\max }(-y)} \mathrm{d} \delta y_{\mathrm{A}} \hat{\mathcal{P}}\left(x_{\mathrm{A}}, \hat{q}(-y) L_{\mathrm{A}}\right) \frac{\mathrm{d} \sigma_{\mathrm{pp}}^{\psi}}{\mathrm{d} y}\left(y+\delta y_{\mathrm{B}}-\delta y_{\mathrm{A}}\right),
$$

where $x_{\mathrm{A}} \equiv e^{\delta y_{\mathrm{A}}}-1, x_{\mathrm{B}} \equiv e^{\delta y_{\mathrm{B}}}-1$, and we used the fact that $\mathrm{d} \sigma_{\mathrm{pp}}^{\psi} / \mathrm{d} y$ is an even function of the rapidity. From (2.11) we can compute the nuclear suppression factor in (minimum bias) heavy-ion collisions,

$$
R_{\mathrm{AB}}(y)=\frac{1}{A B} \frac{\mathrm{d} \sigma_{\mathrm{AB}}^{\psi}}{\mathrm{d} y}(y) / \frac{\mathrm{d} \sigma_{\mathrm{pp}}^{\psi}}{\mathrm{d} y}(y)
$$

Let us note that (2.11) can be rewritten as

$$
\frac{1}{A B} \frac{\mathrm{d} \sigma_{\mathrm{AB}}^{\psi}}{\mathrm{d} y}(y)=\int \mathrm{d} \delta y \hat{\mathcal{P}}_{\mathrm{AB}}(\delta y) \frac{\mathrm{d} \sigma_{\mathrm{pp}}^{\psi}}{\mathrm{d} y}(y+\delta y),
$$

where $\hat{\mathcal{P}}_{\mathrm{AB}}(\delta y)$ is the energy 'loss' $(\delta y>0)$ or 'gain' $(\delta y<0)$ probability distribution in A-B collisions,

$$
\hat{\mathcal{P}}_{\mathrm{AB}}(\delta y) \equiv \int_{0}^{\delta y^{\max }(y)} \mathrm{d} \delta y_{\mathrm{B}} \hat{\mathcal{P}}\left(x_{\mathrm{B}}, \hat{q}(y) L_{\mathrm{B}}\right) \int_{0}^{\delta y^{\max }(-y)} \mathrm{d} \delta y_{\mathrm{A}} \hat{\mathcal{P}}\left(x_{\mathrm{A}}, \hat{q}(-y) L_{\mathrm{A}}\right) \delta\left(\delta y-\delta y_{\mathrm{B}}+\delta y_{\mathrm{A}}\right) .
$$

\subsection{Medium length in the Glauber model}

In minimum bias A-B collisions (i.e., after integration over the impact parameter $\boldsymbol{b}$ ), the effective path length covered by the compact color octet in nucleus A can be calculated in Glauber theory and shown to coincide with an expression derived for minimum bias p-A collisions [6],

$$
\left\langle L_{\mathrm{eff}}^{\mathrm{A}}\right\rangle-L_{\mathrm{p}}=\frac{(A-1)}{A^{2} \rho_{0}} \int \mathrm{d}^{2} s T_{\mathrm{A}}^{2}(\boldsymbol{s}),
$$

and a similar expression for the effective path length in nucleus B. In eq. (2.15) the thickness function $T_{\mathrm{A}}(\boldsymbol{s})$ is normalized as $\int \mathrm{d}^{2} s T_{\mathrm{A}}(\boldsymbol{s})=A$, and we use $L_{\mathrm{p}}=1.5 \mathrm{fm}$ for the length in a proton target and $\rho_{0}=0.17 \mathrm{fm}^{-3}$ for the nuclear density, consistently with [6].

\footnotetext{
${ }^{8}$ Since rescattering processes happen simultaneously in A and B, the result (2.11) should not depend on the order in which the energy losses induced by $\mathrm{A}$ and $\mathrm{B}$ are taken into account. We therefore neglect the shift $\delta y_{\mathrm{B}}$ in $\hat{q}$ and $\delta y^{\max }$ when using (2.6) to go from (2.10) to (2.11). Namely, $\hat{q}\left(-y-\delta y_{\mathrm{B}}\right) \simeq \hat{q}(-y)$ and $\delta y^{\max }\left(-y-\delta y_{\mathrm{B}}\right) \simeq \delta y^{\max }(-y)$. This ensures that $(2.11)$ verifies $\mathrm{d} \sigma_{\mathrm{BA}}(y) / \mathrm{d} y=\mathrm{d} \sigma_{\mathrm{AB}}(-y) / \mathrm{d} y$.
} 
In order to exhibit its centrality dependence, $\psi$ suppression is often measured as a function of the number of participants $\left\langle N_{\text {part }}\right\rangle_{\mathcal{C}}$ corresponding to a given centrality class $\mathcal{C}$, given by [14]

$$
\begin{aligned}
\left\langle N_{\text {part }}\right\rangle_{\mathcal{C}}=\frac{1}{\sigma_{\mathrm{AB}}^{\mathcal{C}}}[ & \int_{\mathcal{C}} \mathrm{d}^{2} \boldsymbol{b} \int \mathrm{d}^{2} \boldsymbol{s} T_{\mathrm{A}}(\boldsymbol{s})\left\{1-\left[1-\frac{\sigma_{\mathrm{NN}}}{B} T_{\mathrm{B}}(\boldsymbol{b}-\boldsymbol{s})\right]^{B}\right\} \\
& \left.+\int_{\mathcal{C}} \mathrm{d}^{2} \boldsymbol{b} \int \mathrm{d}^{2} \boldsymbol{s} T_{\mathrm{B}}(\boldsymbol{s})\left\{1-\left[1-\frac{\sigma_{\mathrm{NN}}}{A} T_{\mathrm{A}}(\boldsymbol{b}-\boldsymbol{s})\right]^{A}\right\}\right] .
\end{aligned}
$$

Here $\sigma_{\mathrm{NN}}$ is the nucleon-nucleon inelastic cross section (we take $\sigma_{\mathrm{NN}}=42 \mathrm{mb}$ at $\sqrt{s}=$ $200 \mathrm{GeV}[15]$ and $\sigma_{\mathrm{NN}}=62.8 \mathrm{mb}$ at $\left.\sqrt{s}=2.76 \mathrm{TeV}[16]\right)$ and $\sigma_{\mathrm{AB}}^{\mathcal{C}}$ is the A-B cross section of that centrality class,

$$
\sigma_{\mathrm{AB}}^{\mathcal{C}}=\int_{\mathcal{C}} \mathrm{d}^{2} \boldsymbol{b}\left\{1-\left[1-\frac{\sigma_{\mathrm{NN}}}{A B} T_{\mathrm{AB}}(\boldsymbol{b})\right]^{A B}\right\},
$$

where $T_{\mathrm{AB}}(\boldsymbol{b}) \equiv \int d^{2} s T_{\mathrm{A}}(\boldsymbol{s}) T_{\mathrm{B}}(\boldsymbol{b}-\boldsymbol{s})$.

In a given centrality class $\mathcal{C}$ of $\mathrm{A}-\mathrm{B}$ collisions, the effective path length of the compact color octet across A can be estimated as

$$
\left\langle L_{\mathrm{eff}}^{\mathrm{A}}\right\rangle_{\mathcal{C}}-L_{\mathrm{p}}=\frac{(A-1)}{A \rho_{0}} \int_{\mathcal{C}} \mathrm{d}^{2} \boldsymbol{b} \int \mathrm{d}^{2} \boldsymbol{s} T_{\mathrm{A}}^{2}(\boldsymbol{s}) T_{\mathrm{B}}(\boldsymbol{b}-\boldsymbol{s}) / \int_{\mathcal{C}} \mathrm{d}^{2} \boldsymbol{b} T_{\mathrm{AB}}(\boldsymbol{b}),
$$

and a similar expression for the path length across B. For the $0-100 \%$ centrality class, i.e., integrating over all impact parameters in eq. (2.18), we recover the minimum bias expression (2.15).

In the following, we will use in (2.11) the length (2.18) to compute $R_{\mathrm{AB}}$ as a function of $\left\langle N_{\text {part }}\right\rangle_{\mathcal{C}}$, eq. (2.16), and the length (2.15) to compute $R_{\mathrm{AB}}$ as a function of $y$ in minimum bias collisions.

\subsection{A simple approximation}

In order to get a baseline of cold nuclear matter effects expected in heavy-ion collisions, a data-driven extrapolation of proton-nucleus measurements has been used at RHIC [17, 18]. Assuming that final-state absorption and $\mathrm{nPDF}$ effects are the dominant cold nuclear effects, it is assumed in these studies that the suppression in A-B collisions is given by that in $\mathrm{p}-\mathrm{A}$ and $\mathrm{p}-\mathrm{B}$ collisions, according to

$$
R_{\mathrm{AB}}(y) \simeq R_{\mathrm{pA}}(-y) \times R_{\mathrm{pB}}(+y),
$$

where $R_{\mathrm{pB}}$ is the cross section ratio,

$$
R_{\mathrm{pB}}(y)=\frac{1}{B} \frac{\mathrm{d} \sigma_{\mathrm{pB}}^{\psi}}{\mathrm{d} y}(y) / \frac{\mathrm{d} \sigma_{\mathrm{pp}}^{\psi}}{\mathrm{d} y}(y) .
$$

Here we show that this approximation also holds in the present energy loss model. Using (2.6) and (2.11) we readily find

$$
\frac{1}{A B} \frac{\mathrm{d} \sigma_{\mathrm{AB}}^{\psi}}{\mathrm{d} y}(y)=\int_{0}^{\delta y^{\max }(-y)} \mathrm{d} \delta y_{\mathrm{A}} \hat{\mathcal{P}}\left(e^{\delta y_{\mathrm{A}}}-1, \hat{q}(-y) L_{\mathrm{A}}\right) R_{\mathrm{pB}}\left(y-\delta y_{\mathrm{A}}\right) \frac{\mathrm{d} \sigma_{\mathrm{pp}}^{\psi}}{\mathrm{d} y}\left(y-\delta y_{\mathrm{A}}\right) .
$$



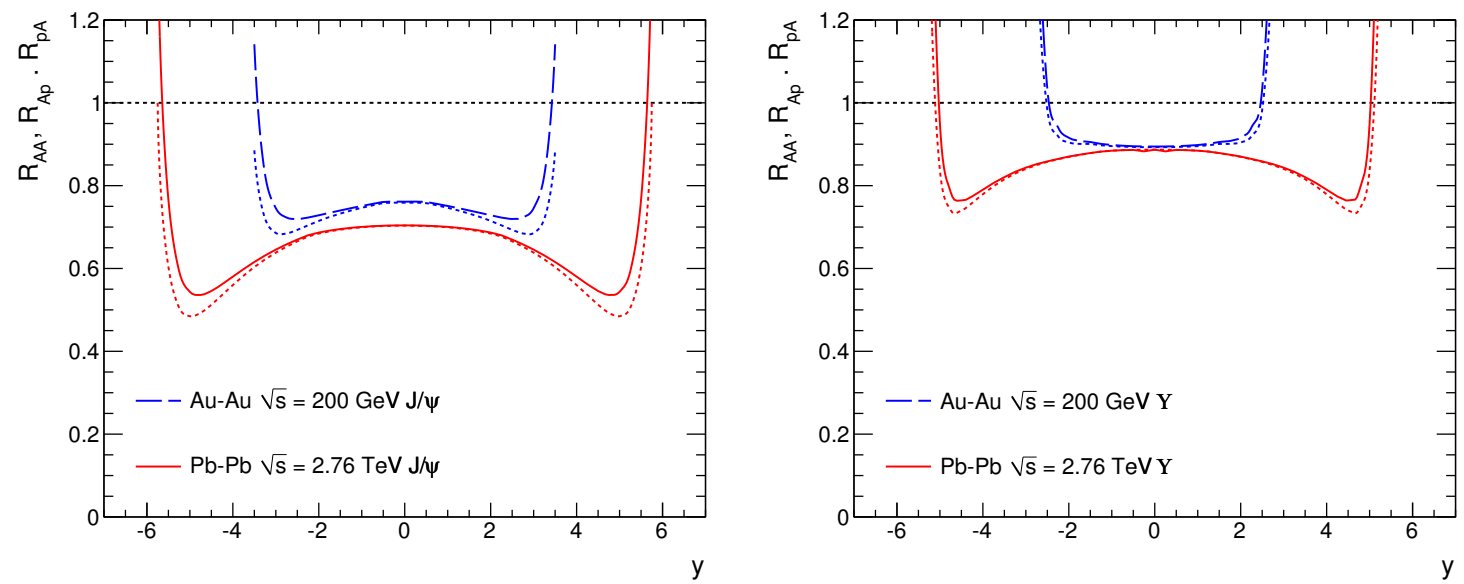

Figure 2. Rapidity dependence of $\mathrm{J} / \psi$ (left) and $\Upsilon$ (right) suppression from coherent energy loss, in minimum bias $\mathrm{Au}-\mathrm{Au}$ collisions at RHIC (long-dashed line) and $\mathrm{Pb}-\mathrm{Pb}$ collisions at LHC (solid) in comparison to the approximation (2.19) (dotted).

Now assuming that $R_{\mathrm{pB}}(y)$ is a much smoother function than $\mathrm{d} \sigma_{\mathrm{pp}}^{\psi} / \mathrm{d} y$, one directly gets

$$
\frac{1}{A B} \frac{\mathrm{d} \sigma_{\mathrm{AB}}^{\psi}}{\mathrm{d} y}(y) \simeq R_{\mathrm{pB}}(y) \int_{0}^{\delta y^{\max }(-y)} \mathrm{d} \delta y_{\mathrm{A}} \hat{\mathcal{P}}\left(e^{\delta y_{\mathrm{A}}}-1, \hat{q}(-y) L_{\mathrm{A}}\right) \frac{\mathrm{d} \sigma_{\mathrm{pp}}^{\psi}}{\mathrm{d} y}\left(-y+\delta y_{\mathrm{A}}\right) .
$$

Using again (2.6), and the definitions (2.12) and (2.20), one directly gets eq. (2.19).

In figure 2 (left) we compute $\mathrm{J} / \psi$ suppression in minimum bias $\mathrm{Au}-\mathrm{Au}$ collisions at RHIC and $\mathrm{Pb}-\mathrm{Pb}$ collisions at LHC using eq. (2.11) and the approximation eq. (2.19). We used $\left\langle L_{\text {eff }}^{\mathrm{A}}\right\rangle=10.21 \mathrm{fm}$ for $\mathrm{A}=\mathrm{Au}$ and $\left\langle L_{\text {eff }}^{\mathrm{A}}\right\rangle=10.11 \mathrm{fm}$ for $\mathrm{A}=\mathrm{Pb}[6]$. A smooth decrease of $R_{\mathrm{AB}}$ is observed as a function of $|y|$, until a value of the rapidity above which $R_{\mathrm{AB}}$ increases rapidly. The rise of $R_{\mathrm{AB}}$ at large rapidity is due to energy 'gain' fluctuations, $\delta y<0$ in (2.13), which dramatically enhance the $\mathrm{J} / \psi$ production cross section, $\sigma_{\mathrm{pp}}^{\psi}(y+\delta y) \gg \sigma_{\mathrm{pp}}^{\psi}(y)$ as $y$ is getting closer to the boundary of phase-space where $\sigma_{\mathrm{pp}}^{\psi}(y)$ becomes vanishingly small. Figure 2 illustrates that the approximation (2.19) proves remarkable. ${ }^{9}$ Let us stress that the increase of $R_{\mathrm{AB}}$ at large $|y|$ seen on figure 2 appears outside the region of validity $|y|<\left|y^{\text {crit }}\right|$ of our model (see section 2.6), and might be spoiled by nuclear absorption effects. However, note that the approximation (2.19) is best precisely in the validity domain of our model. An equally good agreement is observed in the $\Upsilon$ channel, as can be seen in the right panel of figure 2 .

In order to estimate cold nuclear matter effects in $\mathrm{Pb}-\mathrm{Pb}$ collisions at $\sqrt{s}=2.76 \mathrm{TeV}$, the ALICE collaboration determined the product $[8,19]$

$$
R_{\mathrm{Pbp}}\left(y_{\mathrm{lab}}=y-\Delta y, \sqrt{s}=5 \mathrm{TeV}\right) \times R_{\mathrm{pPb}}\left(y_{\mathrm{lab}}=y+\Delta y, \sqrt{s}=5 \mathrm{TeV}\right),
$$

from their $\mathrm{p}-\mathrm{Pb}$ measurements at $\sqrt{s}=5 \mathrm{TeV}$, where $\Delta y=0.465$ (respectively $-\Delta y=$ $-0.465)$ is the boost of the center-of-mass with respect to the laboratory frame in $\mathrm{p}-\mathrm{Pb}$

\footnotetext{
${ }^{9}$ Eq. (2.19) remains quite accurate even when the variation of $R_{\mathrm{pA}}$ with $y$ is fast and the approximation (2.22) is in principle no longer justified.
} 
(respectively $\mathrm{Pb}-\mathrm{p}$ ) collisions. However, we find that the coherent energy loss effects do not follow exactly this extrapolation. In the acceptance of the ALICE muon spectrometer $(2.5 \leq y \leq 4)$, differences range from 10 to $20 \%$.

\subsection{Computing uncertainties}

Equation (2.11) is used to compute $\mathrm{J} / \psi$ and $\Upsilon$ suppression in heavy-ion collisions. It requires the knowledge of the magnitude of the transport coefficient, $\hat{q}_{0}$, as well as the 'slope' $n$ of the p-p cross section, parametrized as $\mathrm{d} \sigma_{\mathrm{pp}}^{\psi} / \mathrm{d} y \propto\left(1-\frac{2 M_{\perp}}{\sqrt{s}} \cosh y\right)^{n} \quad[6] .{ }^{10}$

In order to assess the uncertainties of the model predictions, both quantities are varied around their central value, $S^{0} \equiv\left\{\hat{q}_{0}, n\right\}$. For a given quarkonium state $(\mathrm{J} / \psi$ or $\Upsilon)$ and at a given center-of-mass energy, four predictions are made assuming the following sets of parameters, $S_{1}^{ \pm}=\left\{\hat{q}_{0}^{ \pm}, n\right\}$ and $S_{2}^{ \pm}=\left\{\hat{q}_{0}, n^{ \pm}\right\}$, on top of the central prediction assuming $S^{0}$. The (asymmetric) uncertainties of the model predictions are determined following the prescription suggested in [20],

$$
\begin{aligned}
& \left(\Delta R_{\mathrm{AB}}^{+}\right)^{2}=\sum_{k}\left[\max \left\{R_{\mathrm{AB}}\left(S_{k}^{+}\right)-R_{\mathrm{AB}}\left(S^{0}\right), R_{\mathrm{AB}}\left(S_{k}^{-}\right)-R_{\mathrm{AB}}\left(S^{0}\right), 0\right\}\right]^{2}, \\
& \left(\Delta R_{\mathrm{AB}}^{-}\right)^{2}=\sum_{k}\left[\max \left\{R_{\mathrm{AB}}\left(S^{0}\right)-R_{\mathrm{AB}}\left(S_{k}^{+}\right), R_{\mathrm{AB}}\left(S^{0}\right)-R_{\mathrm{AB}}\left(S_{k}^{-}\right), 0\right\}\right]^{2} .
\end{aligned}
$$

Let us now specify the estimated range for $\hat{q}_{0}$ and $n$. The transport coefficient $\hat{q}_{0}$ is the only free parameter of the model. It is determined by fitting the $\mathrm{J} / \psi$ suppression measured by E866 [21] in p-W over p-Be collisions $(\sqrt{s}=38.7 \mathrm{GeV})$, see [6]. The obtained value is $\hat{q}_{0}=0.075 \pm 0.005 \mathrm{GeV}^{2} / \mathrm{fm}$, with a slightly larger central value, $\hat{q}_{0}=0.087 \mathrm{GeV}^{2} / \mathrm{fm}$, when the $x_{\mathrm{F}}$-range used to perform the fit is reduced to a domain where the E866 data is the most precise. We thus use (in unit $\mathrm{GeV}^{2} / \mathrm{fm}$ ) $\left\{\hat{q}_{0}, \hat{q}_{0}^{-}, \hat{q}_{0}^{+}\right\}=\{0.075,0.070,0.09\}$.

Regarding the slope of the p-p cross section, we shall use at RHIC the values obtained from the fit of the p-p data at $\sqrt{s}=200 \mathrm{GeV}$ given in $[6],\left\{n, n^{-}, n^{+}\right\}=\{8.3,7.2,9.4\}$ for $\mathrm{J} / \psi$ and $\left\{n, n^{-}, n^{+}\right\}=\{6.7,5.7,7.7\}$ for $\Upsilon$. At the LHC, measurements were performed in p-p collisions at $\sqrt{s}=2.76 \mathrm{TeV}$ by the ALICE collaboration [22]. However, those data alone do not allow $n$ to be determined precisely. We therefore rely on p-p data at $\sqrt{s}=7 \mathrm{TeV}$, which give $n=32.3 \pm 7.5$ for $\mathrm{J} / \psi$ and $n=14.2 \pm 2.9$ for $\Upsilon$, and use the following slightly smaller, empirical values $\left\{n, n^{-}, n^{+}\right\}=\{22.5,20,25\}(\mathrm{J} / \psi)$ and $\left\{n, n^{-}, n^{+}\right\}=$ $\{12.5,10,15\}(\Upsilon)$ inferred from the $\sqrt{s}$ dependence of $n$ by a power-law interpolation from RHIC to LHC. It has to be emphasized that the uncertainty coming from the variation of $n$ is subleading with respect to that coming from the variation of $\hat{q}_{0}$, except at large rapidity where the former uncertainty becomes the largest (typically, $y \gtrsim 4$ at LHC).

Another source of uncertainty arises from the feed down of excited states to the inclusive $\mathrm{J} / \psi$ ( or $\Upsilon$ ) production. We however believe that including the effects of excited states would be premature. Indeed, PHENIX [23] and CMS [24] measurements suggest that an additional effect to energy loss plays a role (possibly due to the proximity of excited states with the open flavour threshold) for $\psi^{\prime}$ and $\Upsilon^{\prime}$ production in proton-nucleus collisions at

\footnotetext{
${ }^{10}$ Note that the normalization of the p-p cross section is irrelevant here as it cancels out when computing the factor $R_{\mathrm{AB}}$, eq. (2.12).
} 
RHIC and LHC, respectively. This additional effect is presently not understood, and moreover nothing is known on the magnitude of the possible suppression of $\chi$ states. In those circumstances, the additional uncertainty of our predictions coming from the effect of feed down cannot be quantified here.

\subsection{Range of validity}

The present picture is not expected to hold when the $\psi$ hadronizes in either the projectile nucleus A or target nucleus B and could thus suffer nuclear absorption. Denoting by $t_{\psi}(y)=\left(E(y) / M_{\perp}\right) \cdot \tau_{\psi}$ (where $\tau_{\psi} \simeq 0.3 \mathrm{fm}$ is the $\psi$ proper formation time for both $\mathrm{J} / \psi$ and $\Upsilon$ states [6]) and $t_{\psi}(-y)$ the $\psi$ formation time in the rest frame of $\mathrm{B}$ and $\mathrm{A}$, respectively, hadronization occurs outside each nucleus when $t_{\psi}(y) \gtrsim L_{\text {eff }}^{\mathrm{B}}$ and $t_{\psi}(-y) \gtrsim L_{\mathrm{eff}}^{\mathrm{A}}$. Using eq. (2.3) and $E_{\mathrm{p}} \simeq s /\left(2 m_{\mathrm{p}}\right)$, this condition translates into the following range of validity for the rapidity,

$$
y^{\mathrm{crit}}(\sqrt{s}, B)<y<-y^{\mathrm{crit}}(\sqrt{s}, A) \equiv \ln \left(\frac{\tau_{\psi}}{L_{\mathrm{eff}}^{\mathrm{A}}} \cdot \frac{\sqrt{s}}{2 m_{\mathrm{p}}}\right)
$$

Using $L_{\mathrm{eff}}^{\mathrm{A}} \simeq 10 \mathrm{fm}$ for $\mathrm{A}=\mathrm{Au}$ or $\mathrm{A}=\mathrm{Pb}[6]$, we obtain $y^{\text {crit }} \simeq-1.2$ for $\mathrm{Au}-\mathrm{Au}$ collisions at $\operatorname{RHIC}(\sqrt{s}=200 \mathrm{GeV})$, and $y^{\text {crit }} \simeq-3.8$ for Pb-Pb collisions at $\mathrm{LHC}(\sqrt{s}=2.76 \mathrm{TeV})$. While at LHC the constraint (2.25) should be fulfilled, the PHENIX muon measurements in the rapidity bins $|y| \simeq 2$ lie at the edge of the applicability of the model.

Before RHIC and LHC, an anomalous $\mathrm{J} / \psi$ suppression had been reported by the NA50 experiment in $\mathrm{Pb}-\mathrm{Pb}$ collisions at the SPS $(\sqrt{s}=17 \mathrm{GeV})[25]$. However, $\mathrm{J} / \psi$ hadronization occurs inside one or both nuclei $\left(y^{\text {crit }} \simeq+1.3\right)$ preventing us from making prediction at this energy.

\section{RHIC}

In this section the model predictions on quarkonium suppression in A-B collisions at RHIC are compared to PHENIX and STAR data, as a function of the rapidity (in a given centrality class) in section 3.1, and as a function of $\left\langle N_{\text {part }}\right\rangle$ (in a given rapidity bin) in section 3.2. The interpretation of the differences between the energy loss model predictions and the data is postponed to section 5 .

\subsection{Rapidity dependence}

The rapidity dependence ${ }^{11}$ of $\mathrm{J} / \psi$ suppression is computed in figure 3 (dashed band) in central $\mathrm{Cu}-\mathrm{Cu}$ (top left), $\mathrm{Au}-\mathrm{Au}$ (top right), and $\mathrm{Cu}-\mathrm{Au}$ (bottom) collisions at $\sqrt{s}=$ $200 \mathrm{GeV}$. The suppression is almost independent of the rapidity in the considered range $(|y|<3)$, and slightly more pronounced in Au-Au collisions, $R_{\mathrm{AuAu}} \simeq 0.75$ (at $y=0$ ), than in $\mathrm{Cu}-\mathrm{Cu}$ collisions, $R_{\mathrm{CuCu}} \simeq 0.8$, because of the larger medium length encountered by the $c \bar{c}$ pair in the former collision system. In asymmetric $(\mathrm{Cu}-\mathrm{Au})$ collisions, $R_{\mathrm{AB}}$ is

\footnotetext{
${ }^{11} \mathrm{By}$ convention, in A-B collision the positive rapidity region corresponds to the fragmentation region of nucleus A.
} 


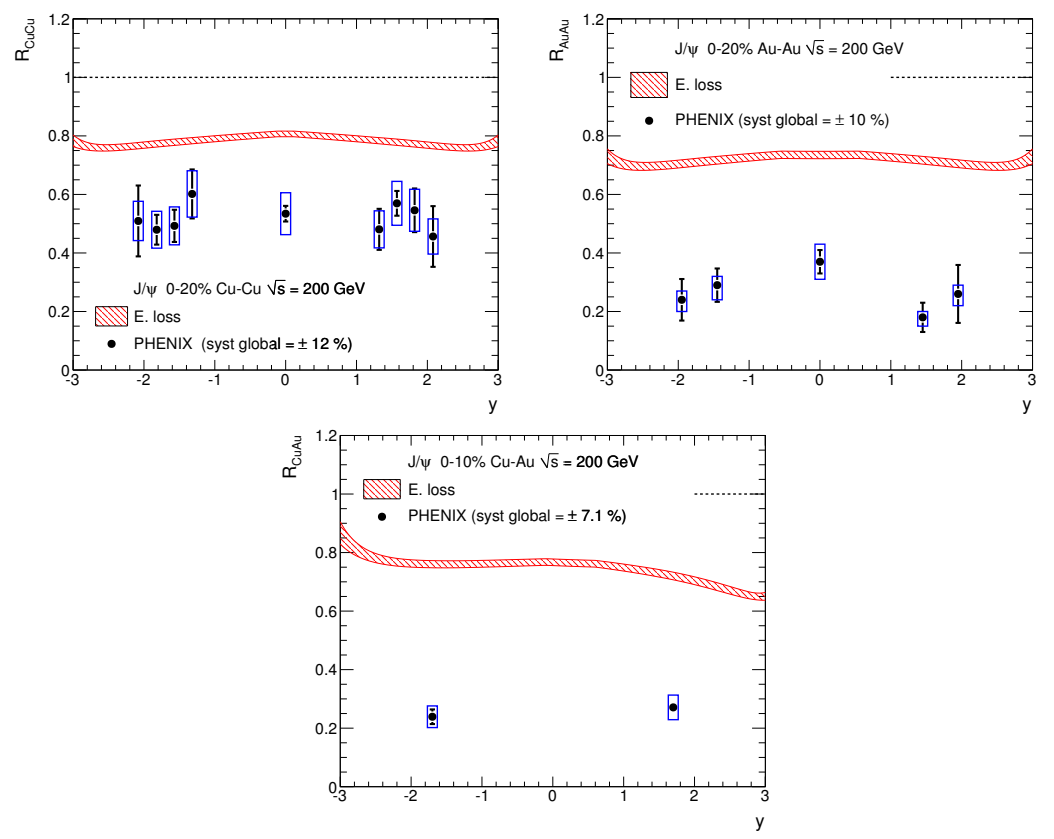

Figure 3. Rapidity dependence of $\mathrm{J} / \psi$ suppression in the $0-20 \%$ most central $\mathrm{Cu}-\mathrm{Cu}$ (top left), $\mathrm{Au}-\mathrm{Au}$ (top right), and $0-10 \%$ most central $\mathrm{Cu}-\mathrm{Au}$ (bottom) collisions at $\sqrt{s}=200 \mathrm{GeV}$ predicted by the energy loss model (dashed band). PHENIX data are from [26-28].

no longer an even function of $y$. At negative rapidity, say $-2<y<0$, the suppression in p-Au (or, d-Au) collisions is rather moderate [6] and the calculations indicate that $R_{\mathrm{pCu}}(y) \simeq R_{\mathrm{pAu}}(y)$. Using (2.19) one thus gets $R_{\mathrm{CuAu}}(y) \simeq R_{\mathrm{CuCu}}(y)$. At positive rapidity, however, the suppression due to coherent energy loss becomes more pronounced, and thus $R_{\mathrm{pAu}}(y)<R_{\mathrm{pCu}}(y)<1$, leading to a stronger suppression in $\mathrm{Cu}-\mathrm{Au}$ with respect to $\mathrm{Cu}-\mathrm{Cu}$ collisions.

The measurements by PHENIX [26-28] are also shown for comparison. ${ }^{12}$ The suppression reported in $\mathrm{Cu}-\mathrm{Cu}$ collisions is significantly more pronounced than the energy loss model prediction. In Au-Au collisions the discrepancy is even more striking: the suppression is $R_{\mathrm{AuAu}} \sim 0.2-0.4$, i.e., 2 to 3 times smaller than the model expectations. Similarly, the PHENIX $\mathrm{Cu}-\mathrm{Au}$ data exhibit a much stronger suppression than that predicted from the sole effect of energy loss. See section 5 for a discussion.

\subsection{Centrality dependence}

Using the relationship between the medium-length and the centrality classes, Eq. (2.18), $\mathrm{J} / \psi$ suppression is determined as a function of $\left\langle N_{\text {part }}\right\rangle$ in $\mathrm{Cu}-\mathrm{Cu}, \mathrm{Au}-\mathrm{Au}$, and $\mathrm{Cu}-\mathrm{Au}$ collisions, see figure 4.

The $\left\langle N_{\text {part }}\right\rangle$ dependence predicted in the model is much less pronounced than the measured one. In $\mathrm{Cu}-\mathrm{Cu}$ and $\mathrm{Au}-\mathrm{Au}$ collisions, a strong discrepancy is seen for $\left\langle N_{\text {part }}\right\rangle \gtrsim 60$ while no significant disagreement is observed for more peripheral collisions. The observed

\footnotetext{
${ }^{12}$ In addition to the PHENIX data, the STAR experiment also performed $\mathrm{J} / \psi$ measurements in $\mathrm{Au}$ - $\mathrm{Au}$ collisions [29], yet with a larger uncertainty.
} 

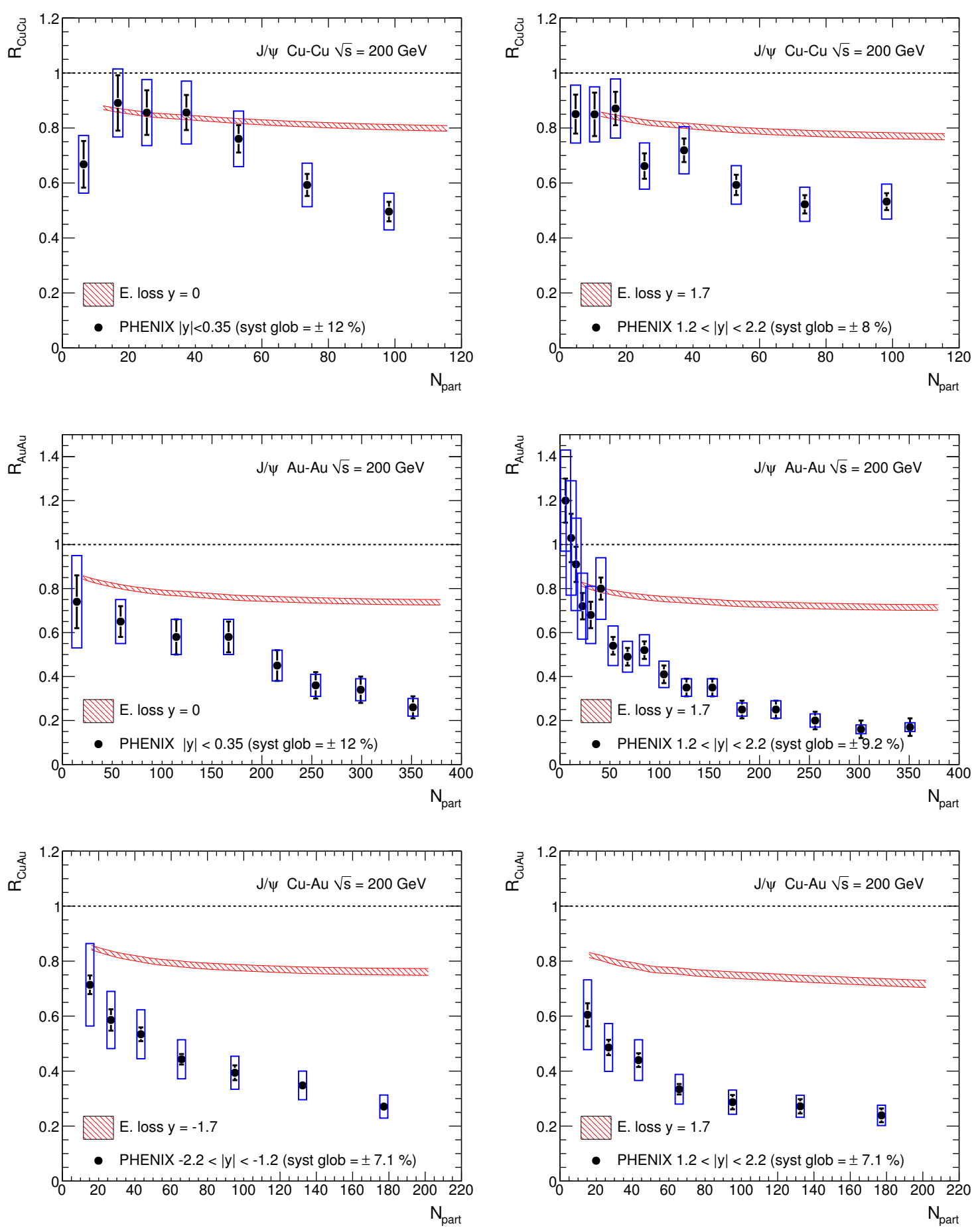

Figure 4. Centrality dependence of $\mathrm{J} / \psi$ suppression in A-B collisions $(\sqrt{s}=200 \mathrm{GeV})$ predicted in the energy loss model (red band). Top row: $\mathrm{Cu}-\mathrm{Cu}$ collisions at $y=0$ (left) and $y=1.7$ (right). Middle row: Au-Au collisions at $y=0$ (left) and $y=1.7$ (right). Bottom row: $\mathrm{Cu}-\mathrm{Au}$ collisions at $y=-1.7$ (left) and $y=1.7$ (right). PHENIX data are from [26-28, 30]. 


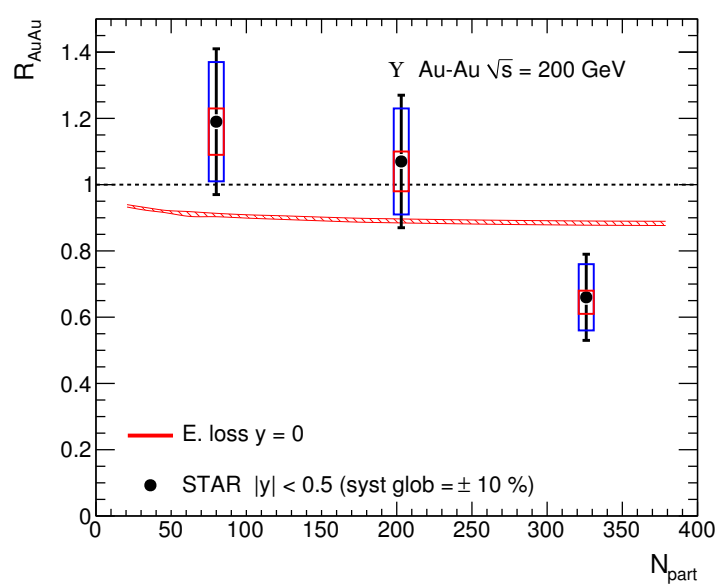

Figure 5. Centrality dependence of $\Upsilon$ suppression in Au-Au collisions $(\sqrt{s}=200 \mathrm{GeV})$ predicted in the energy loss model (red band). STAR data are from [31].

suppression is the strongest in collisions involving the heaviest nucleus $(\mathrm{Au})$. At forward rapidity in $\mathrm{Au}-\mathrm{Au}$ collisions, the model clearly underpredicts the strength of $\mathrm{J} / \psi$ suppression in almost all centrality classes. Despite the fact that the data suffer from a rather large global, systematic uncertainty ranging from $7.1 \%$ to $12 \%$, the reported $\mathrm{J} / \psi$ suppression exceeds significantly the sole effect of parton energy loss in cold nuclear matter. See section 5 for a discussion.

The centrality dependence of $\Upsilon$ suppression is also computed in Au-Au collisions (figure 5) and compared to STAR data [31]. In the most central collisions, the reported $\Upsilon$ suppression is stronger than predicted by the energy loss model.

\section{LHC}

Predictions for $\mathrm{J} / \psi$ (resp. $\Upsilon$ ) suppression in $\mathrm{Pb}-\mathrm{Pb}$ collisions $(\sqrt{s}=2.76 \mathrm{TeV}$ ), arising from coherent energy loss, are given as a function of rapidity (section 4.1) and centrality (section 4.2) and compared to ALICE (resp. CMS) ${ }^{13}$ data.

\subsection{Rapidity dependence}

Figure 6 shows the rapidity dependence of $\mathrm{J} / \psi$ (lower band) and $\Upsilon$ suppression (upper band) expected from energy loss through cold nuclear matter. The different magnitude of the suppression for $\mathrm{J} / \psi$ and $\Upsilon$ arises from the mass dependence of coherent energy loss, see appendix $\mathrm{A}$ and [6]. The quenching factor $R_{\mathrm{PbPb}}$ decreases with rapidity until $|y| \sim 5$, from $R_{\mathrm{PbPb}} \simeq 0.7$ down to $R_{\mathrm{PbPb}} \simeq 0.55$ for $\mathrm{J} / \psi$ and from $R_{\mathrm{PbPb}} \simeq 0.85$ down to $R_{\mathrm{PbPb}} \simeq 0.75$ for $\Upsilon$.

\footnotetext{
${ }^{13}$ Although CMS performed measurements of $\mathrm{J} / \psi$ production in $\mathrm{Pb}-\mathrm{Pb}$ collisions [32], those data are not inclusive in $p_{\perp}$ since a minimal $p_{\perp}$ cut $\left(p_{\perp}^{\text {cut }}=6.5 \mathrm{GeV}\right)$ is applied. It will only be possible to compare the model predictions to these data when addressing the $p_{\perp}$ dependence of $\mathrm{J} / \psi$ suppression in heavy-ion collisions in a future work.
} 


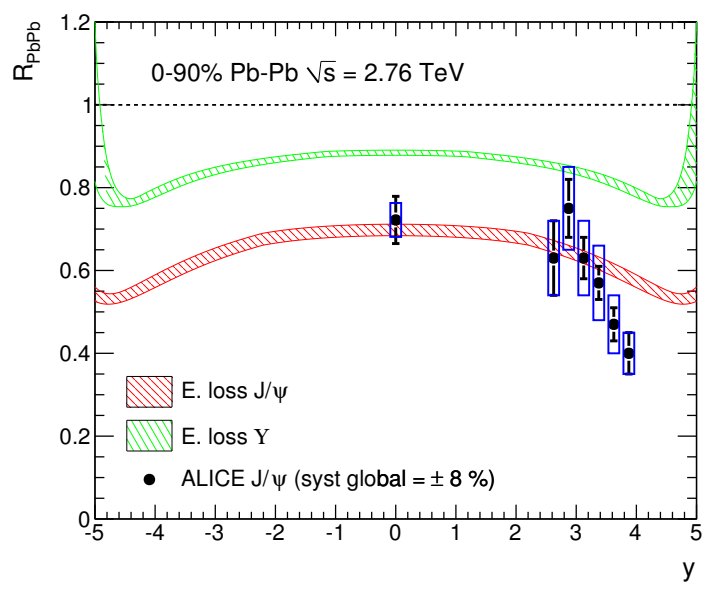

Figure 6. Rapidity dependence of $\mathrm{J} / \psi$ (lower band) and $\Upsilon$ (upper band) suppression in $\mathrm{Pb}-\mathrm{Pb}$ collisions at $\sqrt{s}=2.76 \mathrm{TeV}$. ALICE $\mathrm{J} / \psi$ data are from [33].
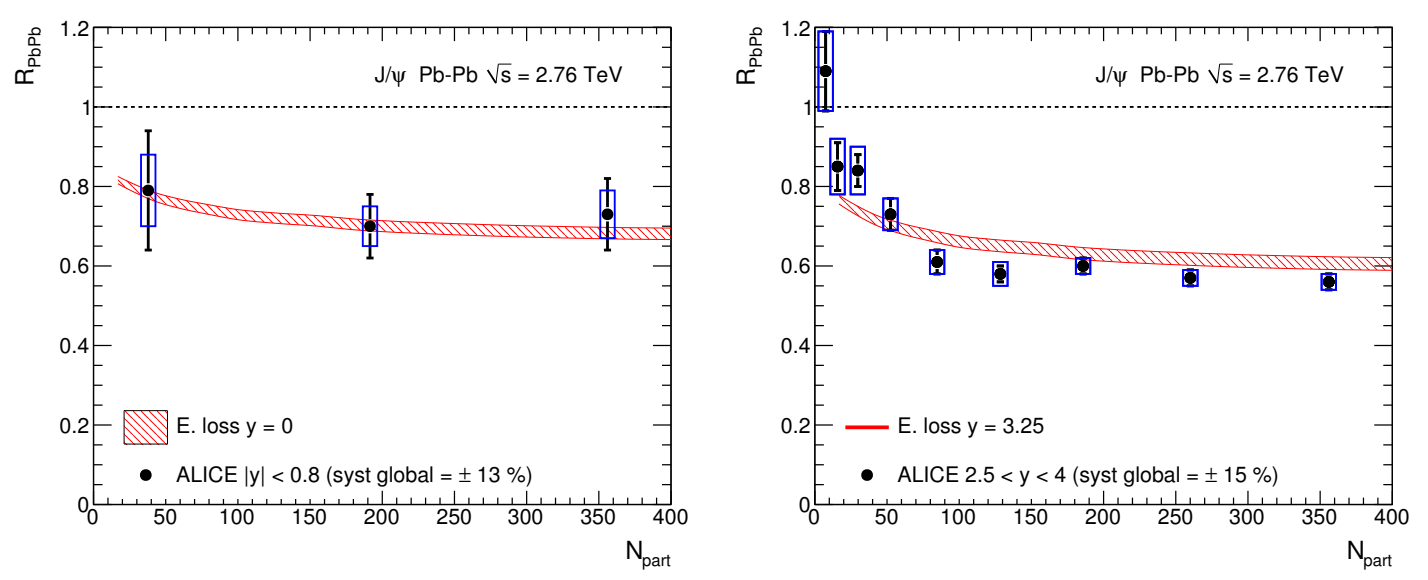

Figure 7. Centrality dependence of $\mathrm{J} / \psi$ suppression in $\mathrm{Pb}-\mathrm{Pb}$ collisions $(\sqrt{s}=2.76 \mathrm{TeV})$ at $y=0$ (left) and $y=3.25$ (right) predicted by the energy loss model. ALICE data are from [33].

The $\mathrm{J} / \psi$ measurements by ALICE follow the same trend as that of the energy loss model, yet with a stronger rapidity dependence at large $y$. The $\mathrm{J} / \psi$ data are well reproduced by the model up to $y \simeq 3.5$, above which the measured suppression is more pronounced.

\subsection{Centrality dependence}

The centrality dependence of $\mathrm{J} / \psi$ suppression due to energy loss is plotted in figure 7 at $y=0$ (left) and $y=3.25$ (right) and compared to ALICE measurements [33]. A good agreement is observed in both rapidity bins, although for $y=3.25$ the $\left\langle N_{\text {part }}\right\rangle$ dependence at low $\left\langle N_{\text {part }}\right\rangle$ is a bit more pronounced in data.

The centrality dependence of $\Upsilon$ suppression is computed in figure 8 at $y=0$ and $y=3.25$ and compared to mid-rapidity $(|y|<2.4)$ CMS data [34]. The suppression is significantly stronger in the data, especially above $\left\langle N_{\text {part }}\right\rangle \gtrsim 200$, for which $R_{\mathrm{PbPb}} \simeq 0.4^{-}$ 


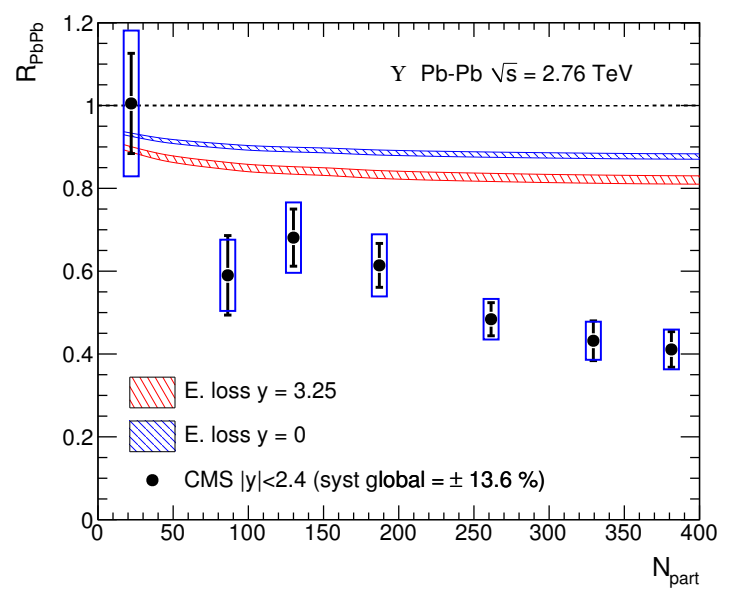

Figure 8. Centrality dependence of $\Upsilon$ suppression in $\mathrm{Pb}-\mathrm{Pb}$ collisions $(\sqrt{s}=2.76 \mathrm{TeV})$ at $y=0$ (upper band) and $y=3.25$ (lower band) predicted by the energy loss model. CMS data are from [34].

0.5 while the model predicts $R_{\mathrm{PbPb}} \simeq 0.9$ at mid-rapidity. This is in sharp contrast with the data-theory comparison in the $\mathrm{J} / \psi$ channel.

\section{Discussion}

At RHIC energy, coherent energy loss leads to a sizable $\mathrm{J} / \psi$ suppression, with a rather flat dependence both in rapidity (figure 3 ) and in centrality (figure 4 ), for instance $R_{\mathrm{AuAu}} \simeq$ $0.7-0.8$ in a broad centrality and rapidity domain. However, as seen in figures 3 and 4 , the predictions of the coherent energy loss model systematically underestimate the strength of $\mathrm{J} / \psi$ suppression observed at RHIC (except in the most peripheral collisions), while excellent agreement is reached in d-Au collisions [6]. Here we must recall that the energy loss model applied to A-B collisions is expected to be valid in the restricted region $|y|<\left|y^{\text {crit }}\right|$, with $y^{\text {crit }} \simeq-1.2$ at RHIC (see section 2.6). Since most of the data points lie in the region $|y| \gtrsim\left|y^{\text {crit }}\right|, \mathrm{J} / \psi$ hadronization might occur inside one of the two nuclei leading to some additional suppression coming from nuclear absorption. It seems however unlikely that nuclear absorption effects could fill the gap between the energy loss model predictions and $\mathrm{Cu}-\mathrm{Au}$ or $\mathrm{Au}-\mathrm{Au}$ data for $\mathrm{J} / \psi$ suppression in this rapidity domain.

We conclude that the large difference between the energy loss model predictions and the data is qualitatively consistent with the onset of hot effects such as Debye screening or gluon dissociation in the hot medium formed in heavy-ion collisions at RHIC. In this respect, it is noticeable in figure 4 that the energy loss expectations are consistent with the data in peripheral collisions, but strongly deviate from them in more central collisions.

At the LHC, a relatively smooth dependence of quarkonium suppression in $y$ or centrality is also predicted in the energy loss model, see figures 6, 7 and 8. However, the dependence of $R_{\mathrm{AA}}$ on rapidity is less flat than at RHIC, with a decrease up to the largest $|y| \sim 3-4$ where the model can be applied $\left(\left|y^{\text {crit }}\right| \simeq 3.8\right.$ at $\sqrt{s}=2.76 \mathrm{TeV}$, see section 2.6). This is at variance with calculations based on $\mathrm{nPDF}$ effects which predict a rise with $|y|$, see for 
instance [35] and figure 9 (right) below. We stress that at LHC the sole effect of energy loss is responsible for a large $\mathrm{J} / \psi$ suppression, $R_{\mathrm{PbPb}} \simeq 0.6-0.7$ in a broad centrality and rapidity domain.

Quite surprisingly, there is a very good agreement between the ALICE data and the energy loss model for $\mathrm{J} / \psi$ suppression in the domain $|y| \lesssim 3$, see figures 6 and 7 . This indicates no net hot medium effect in the $\mathrm{J} / \psi$ channel. In other words, if large hot medium effects are at work in $\mathrm{J} / \psi$ production at $|y| \lesssim 3$, they apparently roughly compensate one another. For instance, the expected $\mathrm{J} / \psi$ suppression from dissociation processes or screening effects in the hot medium might be compensated by the recombination of charm quark pairs. Thus, the energy loss model predictions for $\mathrm{J} / \psi$ suppression in $\mathrm{A}-\mathrm{B}$ collisions are consistent with the presence of recombination at LHC, over a large range of rapidity. At RHIC, the stronger suppression seen in data than in the model indicates that recombination effects, if any, need to be rather small. ${ }^{14}$

In the $\Upsilon$ channel, the suppression measured by CMS is much stronger than the energy loss model prediction, figure 8 . This may be qualitatively understood by the negligible recombination rate of bottom quark pairs when compared to charm quark pairs, leading to some sizable net hot medium effect in the $\Upsilon$ channel.

In the present study we focussed on the effect of energy loss through cold nuclear matter. Of course, the magnitude of the obtained suppression could possibly be affected by additional nPDF or saturation effects. As an illustration, we roughly estimate the magnitude of $\mathrm{J} / \psi$ suppression expected from nPDF effects alone, ${ }^{15}$ estimated using two recent next-to-leading order sets of nuclear parton densities, EPS09 [37] and DSSZ [38]. ${ }^{16}$ (For more calculations involving nPDFs, the reader may refer to [39].) The central EPS09 and DSSZ predictions are shown in figure 9 as thick solid lines. In addition, the calculation has been carried out using the 30 (equally likely) EPS09 error sets coming from the (positive and negative) variation of the 15 parameters used in the global fit analysis. ${ }^{17}$ As can be seen in figure 9 , the bulk of $\mathrm{nPDF}$ calculations points to a moderate $5-15 \% \mathrm{~J} / \psi$ suppression at RHIC. At the LHC, using the DSSZ set leads to less than $5 \%$ suppression, while all but two of the (equally likely) EPS09 error sets predict $R_{\mathrm{PbPb}} \simeq 0.65-0.85$ at $|y| \leq 3$.

At RHIC and the LHC, the expected magnitude of the suppression due to nPDF effects alone never exceeds (and is actually most often smaller than) that due to energy loss, compare for instance figure 3 to figure 9 (left) and figure 6 (red band) to figure 9 (right). At RHIC, energy loss is likely to be more important than nPDF effects. At the LHC, the energy loss effect is likely to be as important (using EPS09) or more important (using

\footnotetext{
${ }^{14}$ This is corroborated by the fact that the number of charm quarks produced in $\mathrm{Au}-\mathrm{Au}$ collisions at RHIC is not too large [3].

${ }^{15}$ Alternatively, one could estimate $\mathrm{J} / \psi$ suppression from saturation effects alone, but we will not do this here. In the case of $\mathrm{p}-\mathrm{Pb}$ collisions at the LHC, we estimated in [6] that when combined with energy loss, the effect of saturation on the magnitude of $\mathrm{J} / \psi$ suppression is quantitatively similar to that of the central set of EPS09 nuclear parton densities. More recent calculations have been proposed in [36].

${ }^{16}$ In this illustration, $\mathrm{nPDF}$ effects are given by $R_{\mathrm{g}}^{\mathrm{Pb}}\left(x_{1}, M_{\perp}^{2}\right) \times R_{\mathrm{g}}^{\mathrm{Pb}}\left(x_{2}, M_{\perp}^{2}\right)$ with the momentum fractions $x_{1,2}=M_{\perp} / \sqrt{s} \times \exp ( \pm y)$.

${ }^{17}$ The variation of each parameter in the EPS09 global fit is such that the $\chi^{2}$ function increases by $\Delta \chi^{2}=50$ from its minimum, corresponding to a $90 \%$ confidence criterion [37].
} 

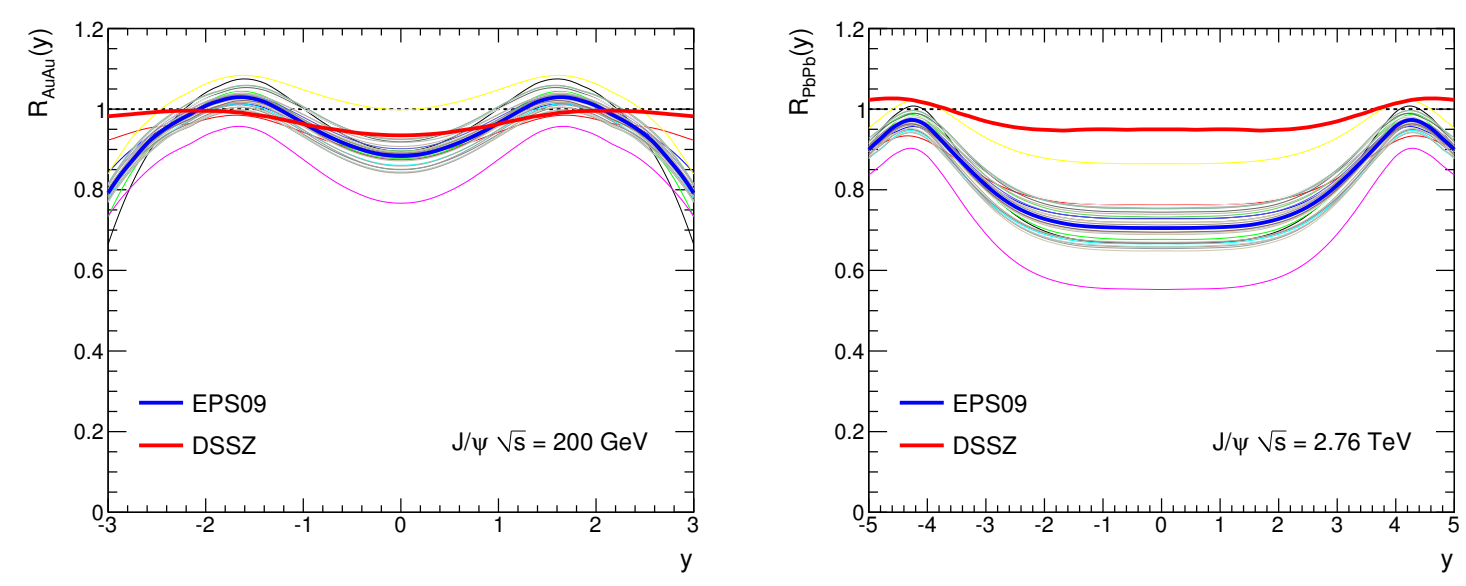

Figure 9. Rapidity dependence of $\mathrm{J} / \psi$ suppression in minimum bias Au-Au collisions at $\sqrt{s}=$ $200 \mathrm{GeV}$ (left) and $\mathrm{Pb}-\mathrm{Pb}$ collisions at $\sqrt{s}=2.76 \mathrm{TeV}$ (right) estimated from the sole effect of nPDFs. The EPS09 and DSSZ central sets are shown as thick solid lines and EPS09 error sets as thin solid lines.

DSSZ) than nPDF effects at mid-rapidity, and more important (for any nPDF choice) at large enough $|y|$. We thus believe our above qualitative discussion to be unaffected by the inclusion of nPDF effects. Our study emphasizes that coherent energy loss effects should be taken into account in order to obtain a reliable baseline for quarkonium suppression in heavy-ion collisions.

Finally, apart from the fact that energy loss is likely to be quantitatively as important (or more) than nPDF effects, we would like to stress that the combined effect of energy loss + nPDF should not be estimated by multiplying the two associated $R_{\mathrm{AA}}$. Indeed, see the case of p-A collisions considered in [6]. It was found there that for consistency, the predictions in a model "energy loss + nPDF" should be made with a different (smaller) value of $\hat{q}_{0}$, obtained from a fit of the "energy loss + nPDF" model to E866 data for $\mathrm{J} / \psi$ suppression in proton-tungsten collisions. The output is that the energy loss predictions for the suppression with or without nPDF effects were of similar magnitude at RHIC and LHC. We expect a similar effect in A-B collisions.

\section{Acknowledgments}

This work is funded by "Agence Nationale de la Recherche" (grant ANR-PARTONPROP).

\section{A Quenching weight}

The quenching weight $\mathcal{P}\left(\varepsilon, E, \ell^{2}\right)$ is related to the radiation spectrum $\mathrm{d} I / \mathrm{d} \varepsilon$ as $[6]$

$$
\mathcal{P}\left(\varepsilon, E, \ell^{2}\right)=\frac{\mathrm{d} I}{\mathrm{~d} \varepsilon} \exp \left\{-\int_{\varepsilon}^{\infty} \mathrm{d} \omega \frac{\mathrm{d} I}{\mathrm{~d} \omega}\right\}=\frac{\partial}{\partial \varepsilon} \exp \left\{-\int_{\varepsilon}^{\infty} \mathrm{d} \omega \frac{\mathrm{d} I}{\mathrm{~d} \omega}\right\}
$$


The medium-induced, coherent radiation spectrum reads ${ }^{18}$

$$
\frac{\mathrm{d} I}{\mathrm{~d} \varepsilon}=\frac{N_{c} \alpha_{s}}{\pi \varepsilon}\left\{\ln \left(1+\frac{\ell^{2} E^{2}}{M_{\perp}^{2} \varepsilon^{2}}\right)-\ln \left(1+\frac{\Lambda_{\mathrm{p}}^{2} E^{2}}{M_{\perp}^{2} \varepsilon^{2}}\right)\right\} \Theta\left(\ell^{2}-\Lambda_{\mathrm{p}}^{2}\right),
$$

where $\ell$ denotes the transverse momentum broadening acquired when crossing the target nucleus $\left(\ell=\ell_{\mathrm{B}}\right.$ for target nucleus $\left.\mathrm{B}\right)$, and $\Lambda_{\mathrm{p}}^{2}=\max \left(\Lambda_{\mathrm{QCD}}^{2}, \ell_{\mathrm{p}}^{2}\right)$ [6]. The medium-induced spectrum (A.2) is defined for a target nucleus B with respect to a target proton, and vanishes when $B=p$.

We easily check that $E \mathcal{P}\left(\varepsilon, E, \ell^{2}\right)$ is a scaling function of $x \equiv \varepsilon / E$ and $\ell^{2}$, and introduce the function $\hat{\mathcal{P}}$,

$$
\hat{\mathcal{P}}\left(x, \ell^{2}\right) \equiv E \mathcal{P}\left(\varepsilon, E, \ell^{2}\right)
$$

Using (A.1), (A.2) and (A.3), we get the explicit form of the 'quenching weight' $\hat{\mathcal{P}}\left(x, \ell^{2}\right)$ to be used in our study,

$$
\begin{aligned}
\hat{\mathcal{P}}\left(x, \ell^{2}\right) & =\frac{N_{c} \alpha_{s}}{\pi x} \ln \left(\frac{x^{2} M_{\perp}^{2}+\ell^{2}}{x^{2} M_{\perp}^{2}+\Lambda_{\mathrm{p}}^{2}}\right) \exp \left\{-\int_{x}^{\infty} \mathrm{d} v \frac{N_{c} \alpha_{s}}{\pi v} \ln \left(\frac{v^{2} M_{\perp}^{2}+\ell^{2}}{v^{2} M_{\perp}^{2}+\Lambda_{\mathrm{p}}^{2}}\right)\right\} \\
& =\frac{\partial}{\partial x} \exp \left\{-\frac{N_{c} \alpha_{s}}{2 \pi} \int_{\Lambda_{\mathrm{p}}^{2} /\left(x^{2} M_{\perp}^{2}\right)}^{\ell^{2} /\left(x^{2} M_{\perp}^{2}\right)} \frac{\mathrm{d} t}{t} \ln (1+t)\right\},
\end{aligned}
$$

where the $\Theta$-function appearing in (A.2) is now implicit. $\hat{\mathcal{P}}$ can be expressed in terms of the dilogarithm $\operatorname{Li}_{2}(x)=-\int_{0}^{x} \frac{\mathrm{d} t}{t} \ln (1-t)$ as

$$
\hat{\mathcal{P}}\left(x, \ell^{2}\right)=\frac{\partial}{\partial x} \exp \left\{\frac{N_{c} \alpha_{s}}{2 \pi}\left[\operatorname{Li}_{2}\left(\frac{-\ell^{2}}{x^{2} M_{\perp}^{2}}\right)-\operatorname{Li}_{2}\left(\frac{-\Lambda_{\mathrm{p}}^{2}}{x^{2} M_{\perp}^{2}}\right)\right]\right\} .
$$

Finally, let us mention that when the transverse momentum broadening $\ell$ becomes very large (i.e., for a very large target nucleus), and $x=\varepsilon / E$ satisfies $\Lambda_{\mathrm{p}}^{2} / M_{\perp}^{2} \ll x^{2} \ll \ell^{2} / M_{\perp}^{2}$, the quenching weight can be approximated by the simple expression (use (A.4))

$$
\hat{\mathcal{P}}\left(x, \ell^{2}\right) \simeq \frac{\partial}{\partial x} \exp \left\{-\frac{N_{c} \alpha_{s}}{\pi} \ln ^{2}\left(\frac{\ell}{x M_{\perp}}\right)\right\} .
$$

For realistic nuclear sizes however, we have $\Lambda_{\mathrm{p}}^{2} \lesssim \ell^{2}$ rather than the strong inequality $\Lambda_{\mathrm{p}}^{2} \ll \ell^{2}$, and the exact expression (A.5) should be preferred to the approximation (A.6).

Open Access. This article is distributed under the terms of the Creative Commons Attribution License (CC-BY 4.0), which permits any use, distribution and reproduction in any medium, provided the original author(s) and source are credited.

\footnotetext{
${ }^{18}$ Note that if another hard colored particle is produced in association with the $Q \bar{Q}$ pair (for instance for $\psi$ produced at large $p_{\perp}$ ) the medium-induced gluon spectrum is similar to (A.2) yet with a different color and kinematical prefactor $[12,13]$.
} 


\section{References}

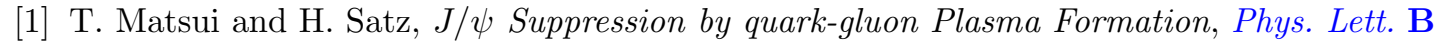
178 (1986) 416 [INSPIRE].

[2] P. Braun-Munzinger and J. Stachel, (Non)thermal aspects of charmonium production and a new look at J/psi suppression, Phys. Lett. B 490 (2000) 196 [nucl-th/0007059] [INSPIRE].

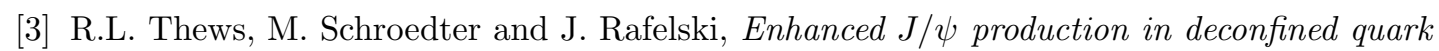
matter, Phys. Rev. C 63 (2001) 054905 [hep-ph/0007323] [INSPIRE].

[4] F. Arleo, S. Peigné and T. Sami, Revisiting scaling properties of medium-induced gluon radiation, Phys. Rev. D 83 (2011) 114036 [arXiv:1006.0818] [INSPIRE].

[5] F. Arleo and S. Peigné, $J / \psi$ suppression in $p$-A collisions from parton energy loss in cold QCD matter, Phys. Rev. Lett. 109 (2012) 122301 [arXiv: 1204.4609] [INSPIRE].

[6] F. Arleo and S. Peigné, Heavy-quarkonium suppression in $p$-A collisions from parton energy loss in cold QCD matter, JHEP 03 (2013) 122 [arXiv: 1212.0434] [INSPIRE].

[7] F. Arleo, R. Kolevatov, S. Peigné and M. Rustamova, Centrality and $p_{\perp}$ dependence of $J / \psi$ suppression in proton-nucleus collisions from parton energy loss, JHEP 05 (2013) 155 [arXiv: 1304.0901] [INSPIRE].

[8] ALICE collaboration, $J / \psi$ production and nuclear effects in $p$-Pb collisions at $\sqrt{S_{N N}}=$ 5.02 TeV, JHEP 02 (2014) 073 [arXiv:1308.6726] [INSPIRE].

[9] LHCb collaboration, Study of $J / \psi$ production and cold nuclear matter effects in $\mathrm{pPb}$ collisions at $\sqrt{s_{N N}}=5 \mathrm{TeV}$, JHEP 02 (2014) 072 [arXiv:1308.6729] [INSPIRE].

[10] P. Hoyer, M. Vänttinen and U. Sukhatme, Violation of factorization in charm hadroproduction, Phys. Lett. B 246 (1990) 217 [INSPIRE].

[11] S. Peigné, F. Arleo and R. Kolevatov, Medium-induced gluon radiation: an update, arXiv: 1402.1671 [INSPIRE].

[12] T. Liou and A.H. Mueller, Parton energy loss in high energy hard forward processes in proton-nucleus collisions, Phys. Rev. D 89 (2014) 074026 [arXiv:1402.1647] [INSPIRE].

[13] S. Peigné and R. Kolevatov, On the process-dependence of coherent medium-induced gluon radiation, arXiv: 1405.4241 [INSPIRE].

[14] A. Bialas, M. Bleszynski and W. Czyz, Multiplicity Distributions in Nucleus-Nucleus Collisions at High-Energies, Nucl. Phys. B 111 (1976) 461 [InSPIRE].

[15] M.L. Miller, K. Reygers, S.J. Sanders and P. Steinberg, Glauber modeling in high energy nuclear collisions, Ann. Rev. Nucl. Part. Sci. 57 (2007) 205 [nucl-ex/0701025] [INSPIRE].

[16] ALICE collaboration, Measurement of inelastic, single- and double-diffraction cross sections in proton-proton collisions at the LHC with ALICE, Eur. Phys. J. C 73 (2013) 2456 [arXiv:1208.4968] [INSPIRE].

[17] R. Granier de Cassagnac, A d-Au data-driven prediction of cold nuclear matter effects on J/psi production in Au-Au collisions at RHIC, J. Phys. G 34 (2007) S955 [hep-ph/0701222] [INSPIRE].

[18] PHENIX collaboration, A. Adare et al., Cold Nuclear Matter Effects on J/Psi as Constrained by Deuteron-Gold Measurements at $\sqrt{s_{N N}}=200$ GeV, Phys. Rev. C 77 (2008) 024912 [Erratum ibid. C 79 (2009) 059901] [arXiv:0711.3917] [InSPIRE]. 
[19] ALICE collaboration, C. Hadjidakis, Quarkonium production in ALICE at the LHC, arXiv: 1405.1177 [INSPIRE].

[20] J. Pumplin, D. Stump, R. Brock, D. Casey, J. Huston et al., Uncertainties of predictions from parton distribution functions. 2. The Hessian method, Phys. Rev. D 65 (2001) 014013 [hep-ph/0101032] [INSPIRE].

[21] NuSea collaboration, M.J. Leitch et al., Measurement of J/psi and psi-prime suppression in p-A collisions at 800-GeV/c, Phys. Rev. Lett. 84 (2000) 3256 [nucl-ex/9909007] [INSPIRE].

[22] ALICE collaboration, Inclusive $J / \psi$ production in pp collisions at $\sqrt{s}=2.76 \mathrm{TeV}$, Phys. Lett. B 718 (2012) 295 [arXiv: 1203.3641] [INSPIRE].

[23] PHENIX collaboration, A. Adare et al., Nuclear Modification of $\psi^{\prime}, \chi_{c}$ and $J / \psi$ Production in $d+A u$ Collisions at $\sqrt{s_{N N}}=200 \mathrm{GeV}$, Phys. Rev. Lett. 111 (2013) 202301 [arXiv: 1305.5516] [INSPIRE].

[24] CMS collaboration, Event activity dependence of $Y(n S)$ production in $\sqrt{s_{N N}}=5.02 \mathrm{TeV} p P b$ and $\sqrt{s}=2.76 \mathrm{TeV}$ pp collisions, JHEP 04 (2014) 103 [arXiv:1312.6300] [INSPIRE].

[25] NA50 collaboration, M.C. Abreu et al., Anomalous J/psi suppression in Pb - Pb interactions at $158 \mathrm{GeV} / \mathrm{c}$ per nucleon, Phys. Lett. B 410 (1997) 337 [INSPIRE].

[26] PHENIX collaboration, A. Adare et al., J/psi Production in $s(N N)^{* *}(1 / 2)=200-G e V$ $\mathrm{Cu}+\mathrm{Cu}$ Collisions, Phys. Rev. Lett. 101 (2008) 122301 [arXiv:0801.0220] [InSPIRE].

[27] PHENIX collaboration, A. Adare et al., $J / \psi$ Production vs Centrality, Transverse Momentum and Rapidity in Au+Au Collisions at $\sqrt{s_{N N}}=200$ GeV, Phys. Rev. Lett. 98 (2007) 232301 [nucl-ex/0611020] [INSPIRE].

[28] PHENIX collaboration, C. Aidala et al., Nuclear matter effects on $J / \psi$ production in asymmetric $\mathrm{Cu}+\mathrm{Au}$ collisions at $\sqrt{s_{N N}}=200 \mathrm{GeV}$, arXiv:1404.1873 [INSPIRE].

[29] STAR collaboration, L. Adamczyk et al., $J / \psi$ production at low $p_{T}$ in $\mathrm{Au}+\mathrm{Au}$ and $\mathrm{Cu}+\mathrm{Cu}$ collisions at $\sqrt{s_{N N}}=200 \mathrm{GeV}$ at STAR, Phys. Rev. C 90 (2014) 024906 [arXiv:1310.3563] [INSPIRE].

[30] PHENIX collaboration, A. Adare et al., $J / \psi$ suppression at forward rapidity in $A u+A u$ collisions at $\sqrt{s_{N N}}=200 \mathrm{GeV}$, Phys. Rev. C 84 (2011) 054912 [arXiv:1103.6269] [INSPIRE].

[31] STAR collaboration, L. Adamczyk et al., Suppression of Upsilon Production in $d+A u$ and Au+Au Collisions at $\sqrt{s_{N N}}=200 \mathrm{GeV}$, Phys. Lett. B 735 (2014) 127 [arXiv:1312.3675] [INSPIRE].

[32] CMS collaboration, Suppression of non-prompt $J / \psi$, prompt $J / \psi$ and $Y(1 S)$ in $\mathrm{PbPb}$ collisions at $\sqrt{s_{N N}}=2.76 \mathrm{TeV}$, JHEP 05 (2012) 063 [arXiv:1201.5069] [INSPIRE].

[33] ALICE collaboration, Centrality, rapidity and transverse momentum dependence of $\mathrm{J} / \psi$ suppression in $\mathrm{Pb}-\mathrm{Pb}$ collisions at $\sqrt{s_{\mathrm{NN}}}=2.76 \mathrm{TeV}$, Phys. Lett. B 734 (2014) 314 [arXiv: 1311.0214] [INSPIRE].

[34] CMS collaboration, Observation of sequential Upsilon suppression in PbPb collisions, Phys. Rev. Lett. 109 (2012) 222301 [arXiv:1208.2826] [INSPIRE].

[35] ALICE collaboration, $J / \psi$ suppression at forward rapidity in $\mathrm{Pb}-\mathrm{Pb}$ collisions at $\sqrt{s_{N N}}=2.76$ TeV, Phys. Rev. Lett. 109 (2012) 072301 [arXiv:1202.1383] [INSPIRE]. 
[36] H. Fujii and K. Watanabe, Heavy quark pair production in high energy $p A$ collisions: Quarkonium, Nucl. Phys. A 915 (2013) 1 [arXiv:1304.2221] [INSPIRE].

[37] K.J. Eskola, H. Paukkunen and C.A. Salgado, EPSO9: A New Generation of NLO and LO Nuclear Parton Distribution Functions, JHEP 04 (2009) 065 [arXiv: 0902.4154] [INSPIRE].

[38] D. de Florian, R. Sassot, P. Zurita and M. Stratmann, Global Analysis of Nuclear Parton Distributions, Phys. Rev. D 85 (2012) 074028 [arXiv:1112.6324] [INSPIRE].

[39] R. Vogt, Cold Nuclear Matter Effects on $J / \psi$ and $\Upsilon$ Production at the LHC, Phys. Rev. C 81 (2010) 044903 [arXiv: 1003.3497] [INSPIRE]. 